\title{
First Synthesis of $\beta$-Keto Sulfoxides by a Palladium-Catalyzed Carbonylative Suzuki Reaction
}

Mercedes Medio-Simón, Cristian Mollar, Nuria Rodríguez and Gregorio Asensio.

\section{Supporting Information}

\section{Table of contents}

$\begin{array}{lr}\text { General methods } & 1 \\ \text { Preparation of starting materials } & 2 \\ \text { Palladium carbonylative Suzuki-Miyaura } & 2 \\ \text { reaction. General Procedure } & 3 \\ \text { NMR data of compounds 3 } & 7 \\ \text { NMR data of compounds 4 } & 8-22 \\ \text { Spectra } & \end{array}$

General methods. Proton magnetic resonance and carbon magnetic resonance were recorded at $300 \mathrm{MHz}$ and $75 \mathrm{~Hz}$ respectively with a Bruker AC-300. Chemical shifts are reported in $\delta$ ppm relative to the $\mathrm{CHCl}_{3}$ peak at $7.20 \mathrm{ppm}\left({ }^{1} \mathrm{H}\right)$ or $77.0 \mathrm{ppm}\left({ }^{13} \mathrm{C}\right)$. High Resolution Mass Spectra were determined on a Fisons VG Autospec instrument. All melting points are uncorrected and were recorded on a Cambridge Instruments apparatus. Reactions were monitored by analytical thin layer chromatography using commercial aluminium sheets pre-coated $\left(0.2 \mathrm{~mm}\right.$ layer thickness) with silica gel $60 \mathrm{~F}_{254}$ (E.Merck). Product purification by flash chromatography was performed using E. Merck Silica Gel (230-400 mesh). 


\section{Preparation of starting materials.}

Synthesis of bromomethyl phenyl sulfoxide $1 .^{1}$ To a solution of methyl phenyl sulfoxide $(10 \mathrm{mmol})$ and anhydrous pyridine $(22 \mathrm{mmol})$ in dry acetonitrile $(40 \mathrm{~mL})$ cooled at $-40^{\circ} \mathrm{C}$ under an argon atmosphere was added dropwise a solution of bromine (20 mmol) in dry acetonitrile $(20 \mathrm{~mL})$ at $-20^{\circ} \mathrm{C}$. The mixture was stirred first at $-40^{\circ} \mathrm{C}$ for $1 \mathrm{~h}$ and then overnight at room temperature. The solvent was evacuated in vacuum and the residue was redissolved in dichloromethane. The organic layer was washed with $\mathrm{Na}_{2} \mathrm{~S}_{2} \mathrm{O}_{3}(0.02 \mathrm{~N}, 30 \mathrm{~mL})$, dried over anhydrous $\mathrm{Na}_{2} \mathrm{SO}_{4}$ and concentrated to dryness. The crude material was purified by flash column chromatography (hexane:ethyl acetate 5:1) to yield sulfoxide 1 (80\%). ${ }^{1} \mathrm{H}-\mathrm{NMR}\left(\cdot 300 \mathrm{MHz}, \mathrm{CDCl}_{3}\right): \delta$ 4.26-4.36 (AB, J= 12.3 $\mathrm{Hz}, 2 \mathrm{H})$ 7.69-7.47 (m, 5H); ${ }^{13} \mathrm{C}-\mathrm{NMR}\left(\cdot 75 \mathrm{MHz}, \mathrm{CDCl}_{3}\right): \delta 49.5$ (t), 125.5 (d), 129.7 (d), 132.5 (d), 141.9 (s); HRMS (E.I.) m/z (M+) calcd. for $\mathrm{C}_{7} \mathrm{H}_{7} \mathrm{BrOS}$ 217.9401, obsd 217.9363.

\section{Palladium catalyzed three component Suzuki-Miyaura reaction with} bromomethyl phenyl sulfoxide. General Procedure.

A mixture of $\alpha$-bromo sulfoxide 1 (0.4 mmol), boronic acid 2 (0.8 mmol) CsF (1.6 mmol) and $\mathrm{Pd}\left(\mathrm{PPh}_{3}\right)_{4}(0.04 \mathrm{mmol})$ was added to a flask fitted with a reflux condenser and a septum inlet. The flask was fluxed with carbon monoxide and then charged with THF (6 mL). The mixture was stirred at $60^{\circ} \mathrm{C}$ under an atmospheric pressure of carbon monoxide. After the appropriate time, the mixture was cooled at room temperature, quenched with water $(10 \mathrm{~mL})$ and extracted with diethyl ether $(2 \times 15 \mathrm{~mL})$ and with dichloromethane ( $3 \times 15 \mathrm{~mL}$ ). The combined organic extracts were dried with $\mathrm{Na}_{2} \mathrm{SO}_{4}$ and evaporated under reduced pressure.

\footnotetext{
${ }^{1}$ Cinquini, M.; Colonna, S. J.Chem. Soc. Perkin 1 1972, 1883.
} 


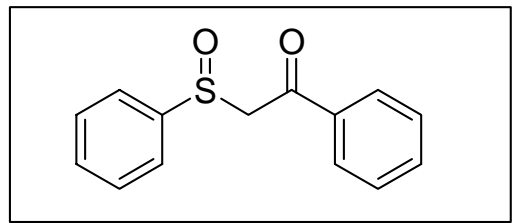

1-phenyl-2-(phenylsulfinyl)ethanone $3 \mathbf{a}^{2}$

Yield: 80\%; m.p.: 68-72 ${ }^{\circ} \mathrm{C}$; ${ }^{1} \mathrm{H}-\mathrm{NMR} \delta 4.29$ (d, J= 14.1 Hz, 1H), 4.45 (d, J= $14.1 \mathrm{~Hz}, 1 \mathrm{H}$ ), 7.41-7.88 (m, 10H);. ${ }^{13}$ C-NMR $\delta 66.0$ (t), 124.2 (d), 128.7 (d+d), 129.3 (d), 131.5 (d), 134.1 (d), 135.9 (s), 143.2 (s), 191.3 (s); HRMS (E.I.) m/z (M ${ }^{+}$) calcd. for $\mathrm{C}_{14} \mathrm{H}_{12} \mathrm{O}_{2} \mathrm{~S} 244.0558$, obsd. 244.0569.<smiles>COc1ccc(C(=O)CS(=O)c2ccccc2)cc1</smiles>

1-(4-methoxyphenyl)-2-(phenylsulfinyl)ethanone $3 \mathbf{b} .^{3}$

Yield: 95\%; m.p.: 89-91 ${ }^{\circ} \mathrm{C} ;{ }^{1} \mathrm{H}-\mathrm{NMR} \delta 3.78$ (s, 3H), 4.16 (d, J= 14 Hz, 1H), 4.44 (d, J= 14 Hz, 1H), 6.83 (d, J= 9 Hz, 1H ), 7.30-7.40 (m, 3H), 7.50-7.60 (m, 2H), 7.80 (d, J=9Hz , 1H); ${ }^{13}$ C-NMR $\delta 55.5$ (q), 65.8 (t), 113.9 (d), 124.1(d), 129.1 (s), 129.2 (d),131.2 (d), 131.4 (d), 143.3 (s), 164.0(s), 190.4(s); HRMS (E.I.) $\mathrm{m} / \mathrm{z}\left(\mathrm{M}^{+}\right)$calcd. for $\mathrm{C}_{15} \mathrm{H}_{14} \mathrm{O}_{3} \mathrm{~S}$ 274.0663, obsd.274.0644.<smiles>COc1cccc(C(=O)CS(=O)c2ccccc2)c1</smiles>

1-(3-methoxyphenyl)-2-(phenylsulfinyl)ethanone 3c. ${ }^{4}$

Yield: 88\%; m.p.: $67-70{ }^{\circ} \mathrm{C} ;{ }^{1} \mathrm{H}-\mathrm{NMR} \delta 3.83$ (s, 3H), 4.27 (d, J= $15 \mathrm{~Hz}, 1 \mathrm{H}$ ), 4.55 (d, J= 14.2 $\mathrm{Hz}, 1 \mathrm{H}), 7.05-7.39$ (m, 4H), 7.40-7.50(m, 3H), 7.60-7.70 (m, 2H); ${ }^{13} \mathrm{C}-\mathrm{NMR} \delta 55.5$ (q), 66.2 (t), 112.5 (d), 120.9 (d), 121.6 (d), 124.3 (d), 129.3 (d), 129.8 (d), 131.6 (d), 137.3 (s), 143.3 (s),160.1 (s), 191.1 (s); HRMS (E.I.) m/z (M+) calcd. for $\mathrm{C}_{15} \mathrm{H}_{14} \mathrm{O}_{3} \mathrm{~S}$ 274.0663, obsd. 274.0669.

\footnotetext{
2 Batigalhia, F.; Zaldini-Hernandes, M.; Ferreira, A. G.; Malvestiti, Ivani; C. Tetrahedron 2001, 57, 9669.

${ }_{3}^{3}$ Olivato, P. R.; Guerrero, S. A.; Zukerman-Schpector, J. Acta Crys. Sect. B 2000, B56(1), 112

${ }^{4}$ Satoh, T.; Fujii, T.; Yamakawa, K Bull. Chem. Soc Jpn 1990, 63, 1266.
} 


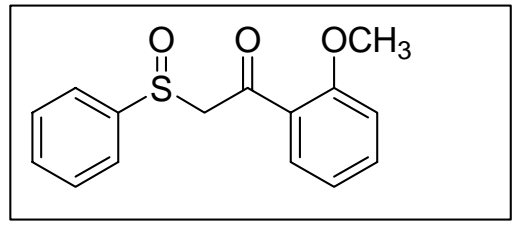

1-(2-methoxyphenyl)-2-(phenylsulfinyl)ethanone 3d.

Yield: 85\%; m.p.: 80-83 ${ }^{\circ} \mathrm{C}$; ${ }^{1} \mathrm{H}-\mathrm{NMR} \delta 3.90$ (s, 3H), 4.44 (d, J= $\left.14.7 \mathrm{~Hz}, 1 \mathrm{H}\right), 4.75$ (d, J= 14.7 Hz, 1H), 6.90-7.05 (m, 2H), 7.47-7.54(m, 4H), 7.68-7.74 (m, 3H); ${ }^{13} \mathrm{C}-\mathrm{NMR} \delta 55.6$ (q), 71.2 (t), 111.6 (d), 121.0 (d), 124.6 (d), 126.0 (s), 129.1 (d), 131.0 (d), 131.2 (d), 135.2 (d), 144.0 (s),159.1 (s), 192.0 (s); HRMS (E.I.) m/z (M $\mathrm{M}^{+}$) calcd. for $\mathrm{C}_{15} \mathrm{H}_{14} \mathrm{O}_{3} \mathrm{~S}$ 274.0663, obsd. 274.0667 .<smiles>Cc1ccccc1C(=O)CS(=O)c1ccccc1</smiles>

1-(2-methylphenyl)-2-(phenylsulfinyl)ethanone 3e.

Yield: 88\%; oil; ${ }^{1} \mathrm{H}-\mathrm{NMR} \delta 2.47$ (s, 3H), 4.27 (d, J= 14.5 Hz, 1H), 4.56 (d, J= 14.5 Hz, 1H), 7.23-7.28 (m, 2H), 7.41 (t, J= 7.8 Hz, 1H), 7.50-7.52 (m, 3H), 7.64 (d, J= 7.8 Hz, 1H), 7.697.72 (m, 2H); ${ }^{13} \mathrm{C}-\mathrm{NMR} \delta 21.5$ (q), 68.2 (t), 124.2 (d), 125.8 (d), 129.3 (d), 129.9 (d),131.5 (d), 132.2 (d), 132.6 (d), 135.5 (s), 139.7 (s), 143.2 (s), 193.9 (s); HRMS (E.I.) m/z (M calcd. for $\mathrm{C}_{15} \mathrm{H}_{14} \mathrm{O}_{2} \mathrm{~S}$ 258.0715, obsd. 258.0719.

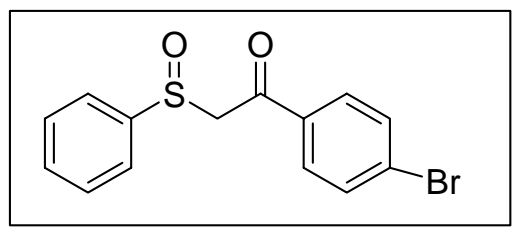

1-(4-bromophenyl)-2-(phenylsulfinyl)ethanone $3 \mathbf{f}^{5}$

Yield: 54\%; m.p.: 90-94 ${ }^{\circ} \mathrm{C}$; ${ }^{1} \mathrm{H}-\mathrm{NMR} \delta 4.26$ (d, J= $\left.14.1 \mathrm{~Hz}, 1 \mathrm{H}\right), 4.50$ (d, J= 15 Hz, 1H), 7.50-7.75 (m, 9H);. ${ }^{13}$ C-NMR $\delta 65.6$ (t), 124.2 (d), 129.4 (d), 129.7 (d), 130.3 (d), 131.7 (d), 132.1 (s), 134.8 (s), 142.9 (s), 190.4 (s);. HRMS (E.I.) m/z (M $)$ calcd. for $\mathrm{C}_{14} \mathrm{H}_{11} \mathrm{BrO}_{2} \mathrm{~S}$ 321.9663, obsd. 321.9655

\footnotetext{
${ }^{5}$ Issa, Y. M.; Etaiw, S. H.; El Ansary, A. L. Indian J. Chem., Sect. A: 1979, 18A, 480.
} 


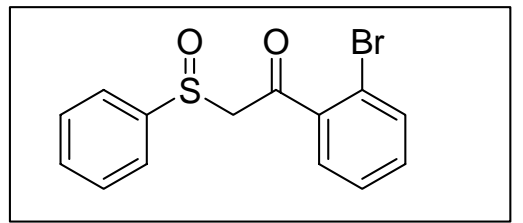

1-(2-bromophenyl)-2-(phenylsulfinyl)ethanone 3g.

Yield: 45\%; oil; ${ }^{1} \mathrm{H}-\mathrm{NMR} \delta 4.40$ (d, J= 14.4 Hz, 1H), 4.57 (d, J= 14.4 Hz, 1H), 7.31-7.71 (m, 9H); ${ }^{13}$ C-NMR $\delta 68.6$ (t), 119.4 (s), 124.3 (d), 127.7 (d), 129.4 (d), 130.0 (d), 131.6 (d), 132.8 (d), 133.7 (d), 139.5 (s), 143.0 (s), 194.31 (s); HRMS (E.I.) m/z ( $\mathrm{M}^{+}$) calcd. for for $\mathrm{C}_{14} \mathrm{H}_{11} \mathrm{BrO}_{2} \mathrm{~S}$ 321.9663, obsd. 321.9657.

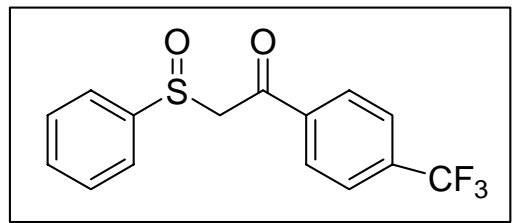

2-(phenylsulfinyl)-1-(4-(trifluoromethyl)phenyl)ethanone $\mathbf{3 h}$.

Yield: 77\%; m.p.: 128-131 ${ }^{\circ} \mathrm{C} ;{ }^{1} \mathrm{H}-\mathrm{NMR} \delta 4.33$ (d, J= 13.9 Hz, 1H), 4.52 (d, J= 13.9 Hz, 1H), 7.43-7.44 (m, 3H), 7.59-7.61 (m, 2H), 7.65 (d, J= 8.1 Hz, 1H); 7.93 (d, J= 8.1 Hz, 1H); ${ }^{13} \mathrm{C}-$ NMR $\delta 65.6$ (t), 123.3 (q, J= 271.8 Hz), 124.1 (d), 125.8 (d), 129.2 (d), 129.4 (d), 131.8 (d), 135.2 (q, J= $32.9 \mathrm{~Hz}$ ), 138.7 (s), 142.8 (s), 190.6 (s); HRMS (E.I.) m/z (M ${ }^{+}$) calcd. for $\mathrm{C}_{15} \mathrm{H}_{11} \mathrm{O}_{2} \mathrm{~F}_{3} \mathrm{~S}$ 312.0431, obsd. 312.0434.

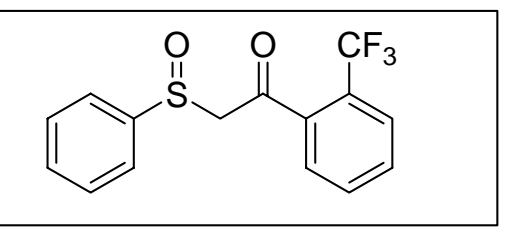

2-(phenylsulfinyl)-1-(2-(trifluoromethyl)phenyl)ethanone 3i.

Yield: 33\%; m.p.:70-74 ${ }^{\circ} \mathrm{C}$; ${ }^{1} \mathrm{H}-\mathrm{NMR} \delta, 4.19$ (d, J= $14.7 \mathrm{~Hz}, 1 \mathrm{H}$ ), 4.41 (d, J= $14.7 \mathrm{~Hz}$, 1H),7.52-7.73 (m, 9H); ${ }^{13} \mathrm{C}-\mathrm{NMR} \delta 69.2$ (t), 123.3 (q, J= $272 \mathrm{~Hz}$ ), 124.2 (d), 125.1 (d), 127.8 (d), 129,5 (d), 131.1 (d), 131.7 (d), 132.1 (d), 138.1 (s), 142.9 (s), 194.6 (s); HRMS (E.I.) $\mathrm{m} / \mathrm{z}\left(\mathrm{M}^{+}\right)$calcd. for $\mathrm{C}_{15} \mathrm{H}_{11} \mathrm{O}_{2} \mathrm{~F}_{3} \mathrm{~S}$ 312.04318, obsd. 312.0437. 
<smiles>O=C(CS(=O)c1ccccc1)c1cccc([N+](=O)[O-])c1</smiles>

1-(3-nitrophenyl)-2-(phenylsulfinyl)ethanone 3k .

Yield: 55\%; m.p.: 118-120 ${ }^{\circ} \mathrm{C} ;{ }^{1} \mathrm{H}-\mathrm{NMR} \delta 4.38$ (d, J= $\left.13.8 \mathrm{~Hz}, 1 \mathrm{H}\right), 4.50$ (d, J= $\left.13.8 \mathrm{~Hz}, 1 \mathrm{H}\right)$, 7.49-7.51 (m, 3H), 7.64-7.69 (m, 3H), 8.23 (d, J=7.8 Hz, 1H) 8.41 (dd, J= 7.8 and $1.2 \mathrm{~Hz}$, 1H), 8.63(d, J=1.2 Hz,1H), ${ }^{13} \mathrm{C}-\mathrm{NMR} \delta 65.0$ (t), 123.6 (d), 124.0 (d), 128.1 (d), 129.5(d), 130.1 (d), 131.8 (d), 134.3 (d), 137.4 (s), 142.4 (s), 148.3 (s), 189.4 (s); HRMS (E.I.) m/z $\left(\mathrm{M}^{+}\right)$calcd. for $\mathrm{C}_{14} \mathrm{H}_{11} \mathrm{NO}_{4} \mathrm{~S} 289.0408$, obsd. 289.0410.

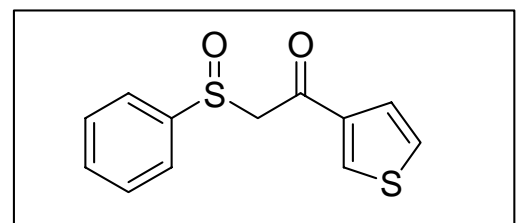

2-(phenylsulfinyl)-1-(3-thienyl)ethanone 31.

Yield: 99\%; m.p.: 90-94 ${ }^{\circ} \mathrm{C}$; ${ }^{1} \mathrm{H}-\mathrm{NMR} \delta 4.10$ (d, J= 13.5 Hz, 1H), 4.34 (d, J= $13.5 \mathrm{~Hz}, 1 \mathrm{H}$ ), 7.23 (dd, J=5.1 and 2.8 Hz, 1H), 7.40-7.44 (m, 4H), 7.58-7.61 (m, 2H), 8.00 (dd, 2.8 and 0.9 $\mathrm{Hz}, 1 \mathrm{H}) ;{ }^{13} \mathrm{C}-\mathrm{NMR} \delta 66.8$ (t), 124.1 (d), 126.8 (d), 126.9 (d), 129.3 (d), 131.5 (d), 134.8(d), 141.5 (s), 143.0 (s), 184.9 (s); HRMS (E.I.) m/z (M ${ }^{+}$) calcd. for $\mathrm{C}_{12} \mathrm{H}_{10} \mathrm{O}_{2} \mathrm{~S}_{2}$ 250.0122, obsd. 250.0110 .<smiles>O=C(CS(=O)c1ccccc1)c1cccs1</smiles>

2-(phenylsulfinyl)-1-(2-thienyl)ethanone $3 \mathbf{m}^{6}$

Yield: 72\%; oil; ${ }^{1} \mathrm{H}-\mathrm{NMR} \delta 4.18$ (d, J=13.3 Hz, 1H), 4.43 (d, J=13.3 Hz, 1H), 7.11 (dd J= 4.7 and $4 \mathrm{~Hz}, 1 \mathrm{H}), 7.48-7.51(3 \mathrm{H}), 7.67-7.71(\mathrm{~m}, 4 \mathrm{H}) ;{ }^{13} \mathrm{C}-\mathrm{NMR} \delta, 66.3$ (t), 124.2 (d), 128.5 (d), 129.3 (d), 131.7 (d), 134.4 (d), 135.9 (d), 143.0 (s), 143.6 (s), 183.3 (s); HRMS (E.I.) m/z $\left(\mathrm{M}^{+}\right)$calcd. for $\mathrm{C}_{12} \mathrm{H}_{10} \mathrm{O}_{2} \mathrm{~S}_{2}$ 250.0122, obsd.250.0110.

\footnotetext{
${ }^{6}$ Kunick, C.; Messinger, P. Chem. Ber. 1986, 119, 1429.
} 


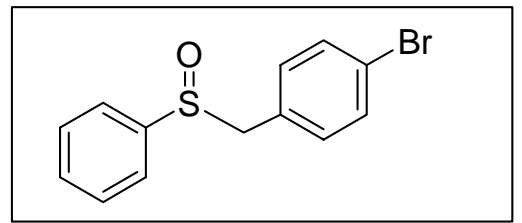

(4-bromobenzyl)phenyl sulfoxide $\mathbf{4 f}^{7}$

Yield: 9\%; m.p.: 155-159 ${ }^{\circ} \mathrm{C}{ }^{1} \mathrm{H}-\mathrm{NMR}\left(\mathrm{CDCl}_{3}\right): \delta$ 3.92-4.02 (AB, 2H, J= $\left.12.7 \mathrm{~Hz}\right), 6.82(\mathrm{~d}$, $\mathrm{J}=8.4 \mathrm{~Hz}, 2 \mathrm{H}), 7.35-7.47(\mathrm{~m}, 7 \mathrm{H}) ;{ }^{13} \mathrm{C}-\mathrm{NMR}\left(\mathrm{CDCl}_{3}\right): \delta 62.4(\mathrm{t}), 122.6(\mathrm{~s}), 124.3(\mathrm{~d}), 127.9$ (s), 129.0 (d), 131.3 (d), 131.5 (d), 131.9 (d), 142.3 (s); HRMS: calcd for $\mathrm{C}_{13} \mathrm{H}_{11} \mathrm{BrOS}$ 293.9714 found 293.9714 .

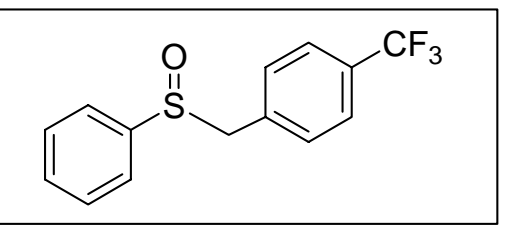

(4-trifluoromethylbenzyl)phenyl sulfoxide $\mathbf{4 h} \mathbf{h}^{8}$

Yield: 23\%; m.p.: $141^{\circ} \mathrm{C}$ (subl.); ${ }^{1} \mathrm{H}-\mathrm{NMR}\left(\mathrm{CDCl}_{3}\right): \delta 3.99-4.13(\mathrm{AB}, 2 \mathrm{H}, \mathrm{J}=12.7 \mathrm{~Hz}), 7.08$ (d, J= 8.1Hz, 2H), 7.35-7.51 (m, 7H); ${ }^{13} \mathrm{C}-\mathrm{NMR}\left(\mathrm{CDCl}_{3}\right): \delta 62.5(\mathrm{t}), 123.9(\mathrm{q}, \mathrm{J}=272.1)$, 124.2 (d), 125.2 (q, J= 3.7 Hz), 129.0 (d), 130.5 (q, J= 26.7), 130.6 (d), 131.4 (d), 133.0 (d), 142.2 (s); HRMS: calcd for $\mathrm{C}_{14} \mathrm{H}_{11} \mathrm{~F}_{3} \mathrm{OS} 284.0483$ found 284.0479 .

\footnotetext{
${ }^{7}$ Rodríguez, N.; Cuenca, A.; Ramírez de Arellano, C.; Medio-Simón, M.; Peine, D.; Asensio, G. J. Org. Chem. 2004, 69, 8070.

${ }^{8}$ Kersten, M.; Wenschuh, E. Phosphorus, Sulfur and Silicon and the Related Elements 1993, 80(1-4), 81.
} 
<smiles>O=S(CBr)c1ccccc1</smiles>

Bromomethyl phenyl sulfoxide $\mathbf{1}$

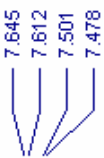

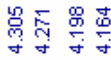
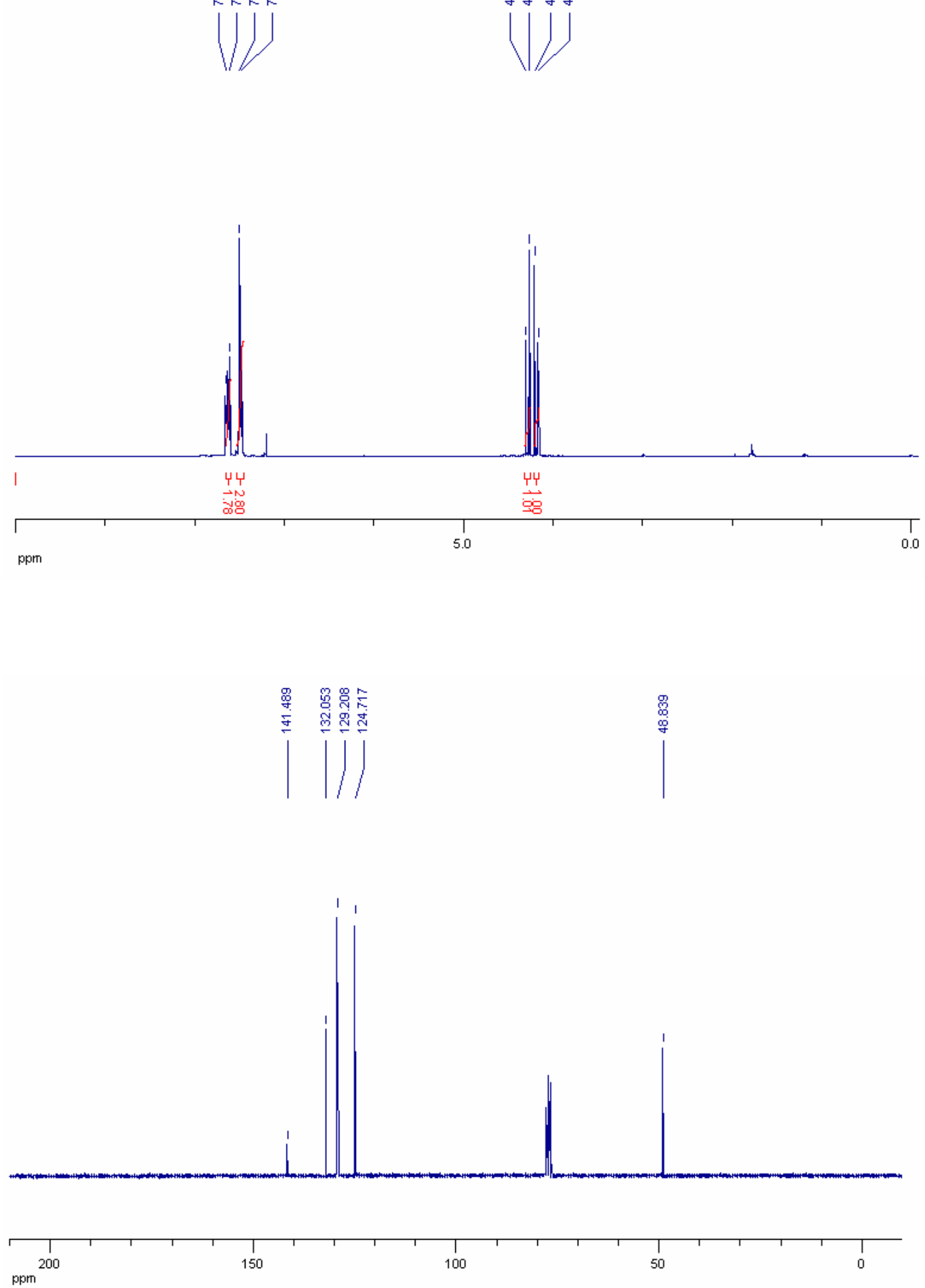


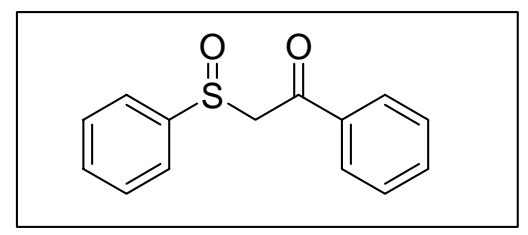

1-phenyl-2-(phenylsulfinyl)ethanone 3a.
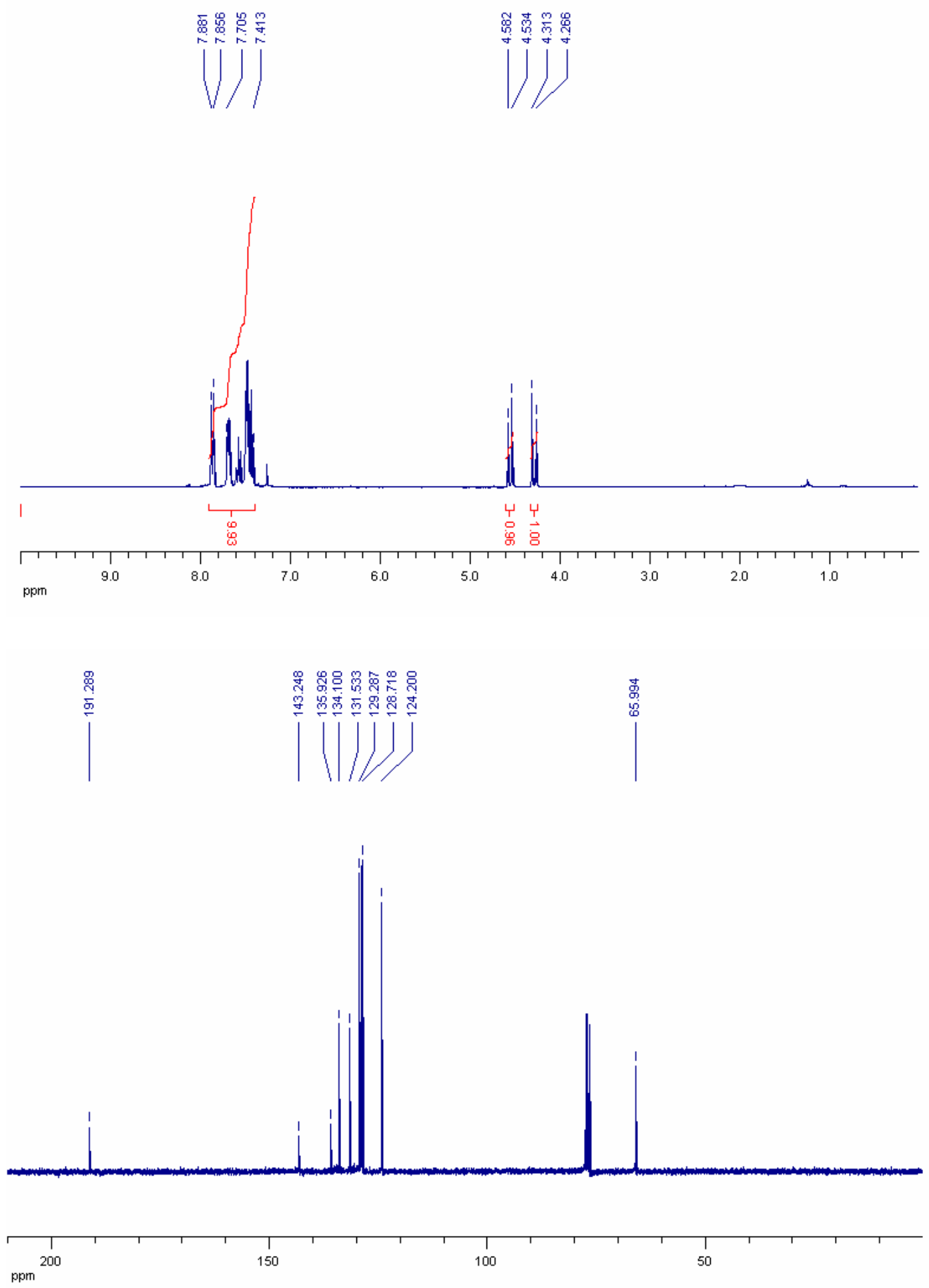

9 


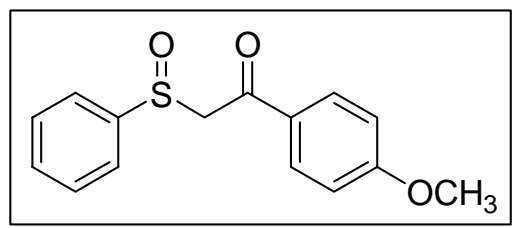

1-(4-methoxyphenyl)-2-(phenylsulfinyl)ethanone 3b.
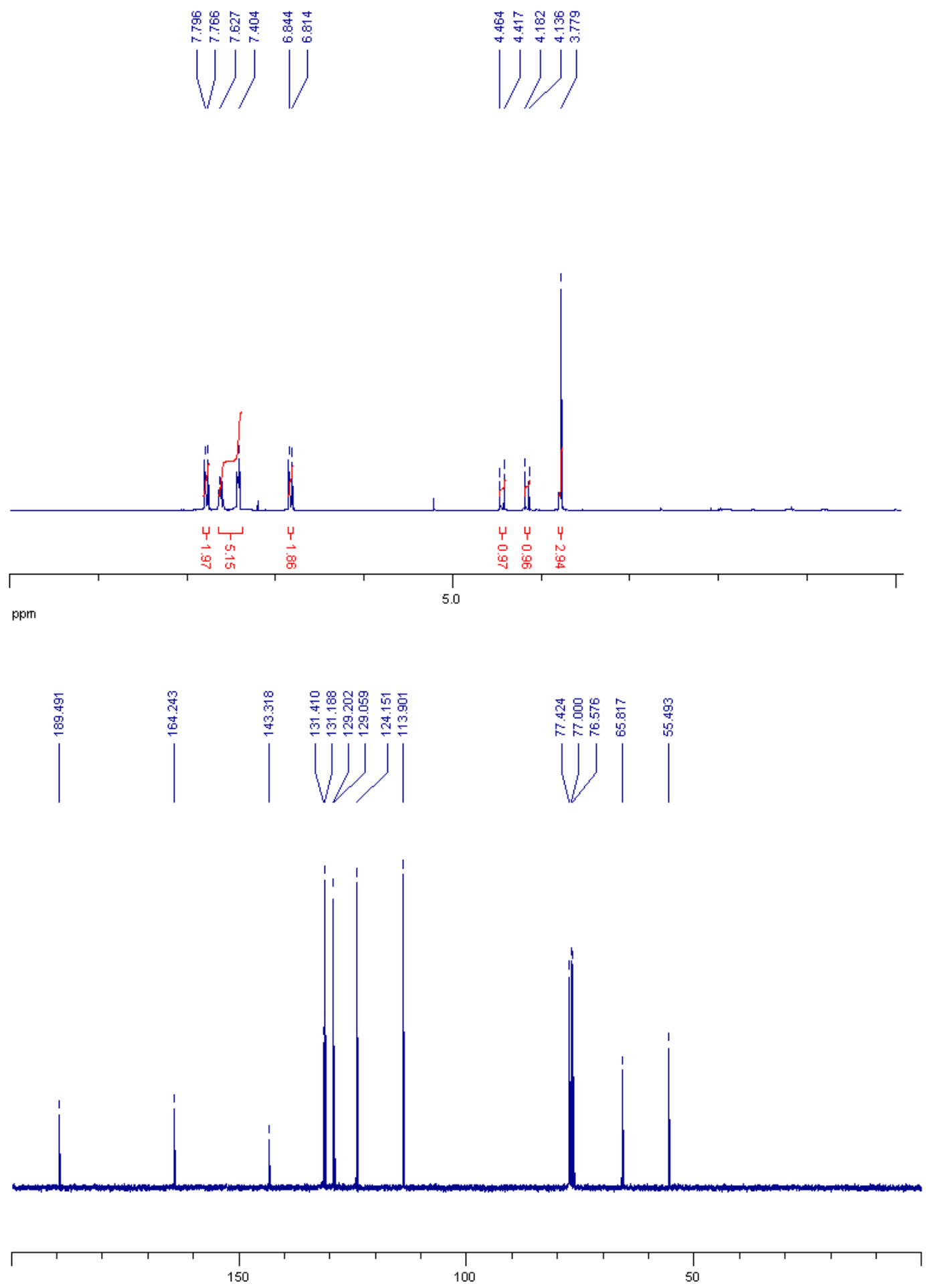
<smiles>COc1cccc(C(=O)CS(=O)c2ccccc2)c1</smiles>

1-(3-methoxyphenyl)-2-(phenylsulfinyl)ethanone 3c

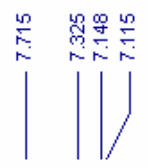

$\mid$
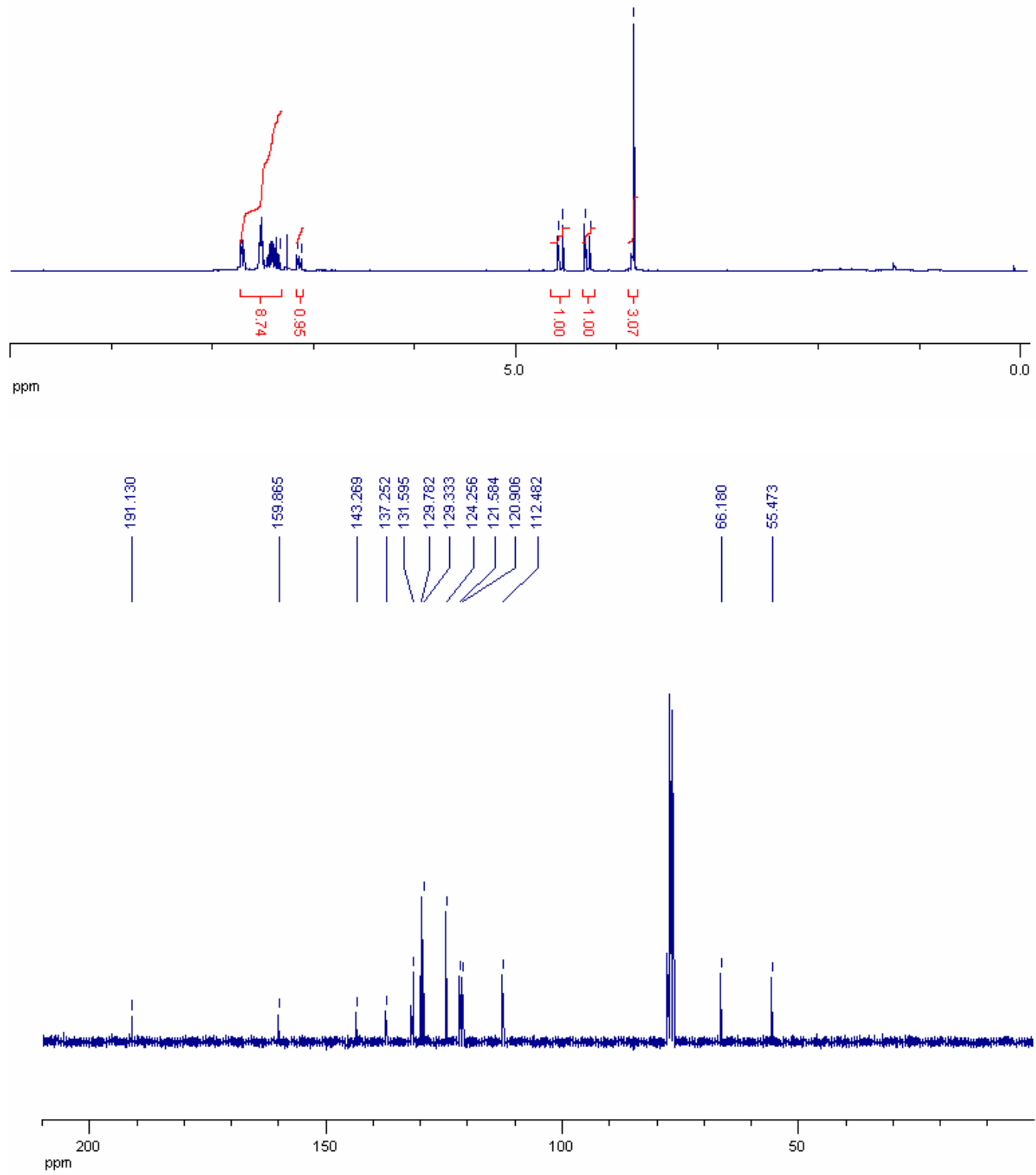


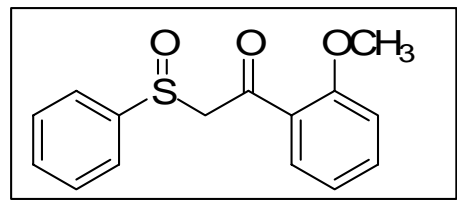

1-(2-methoxyphenyl)-2-(phenylsulfinyl)ethanone 3d.
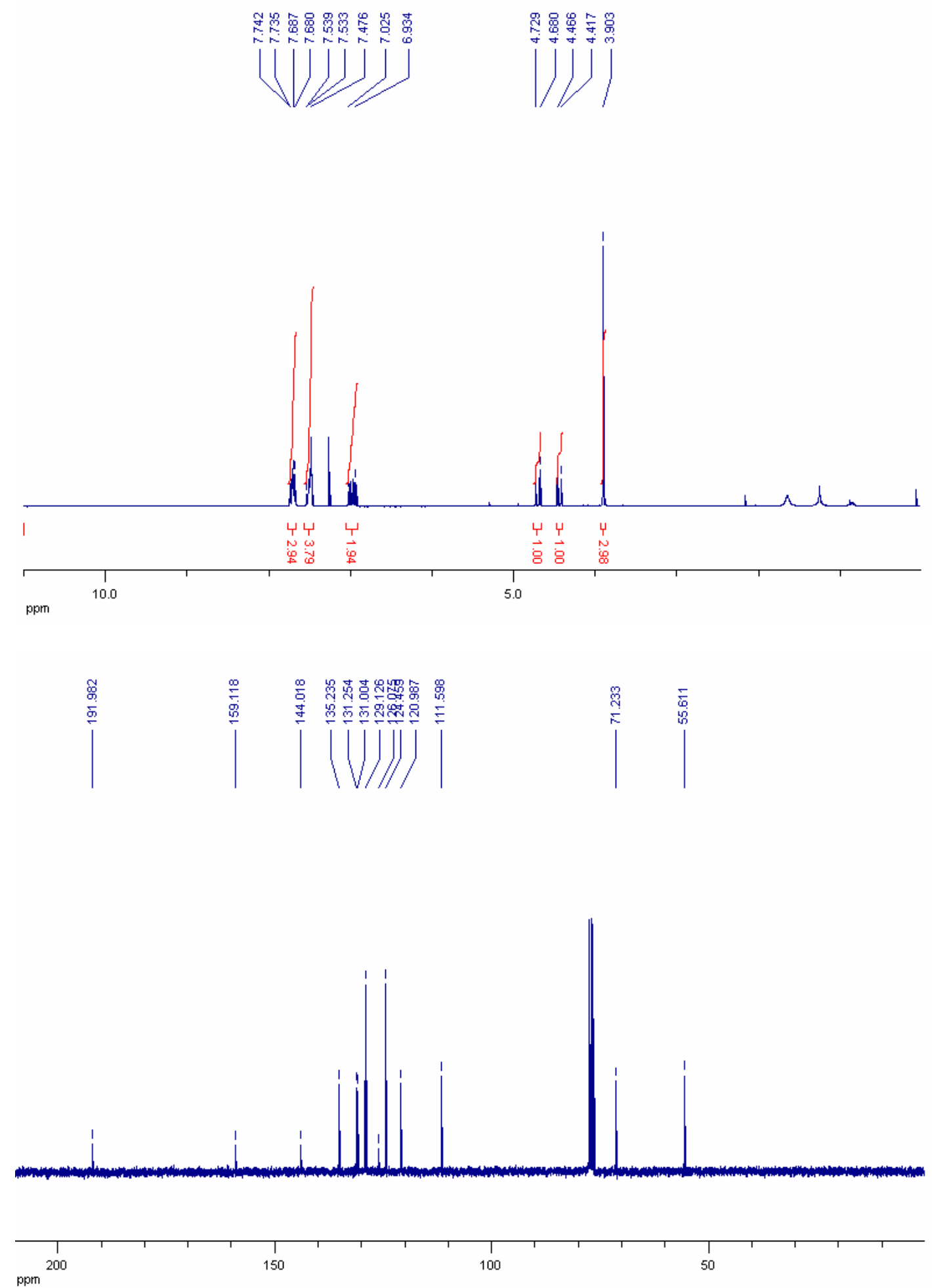


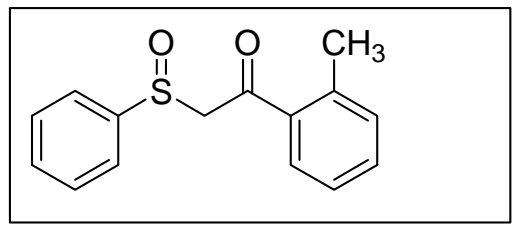

1-(2-methylphenyl)-2-(phenylsulfinyl)ethanone 3e.
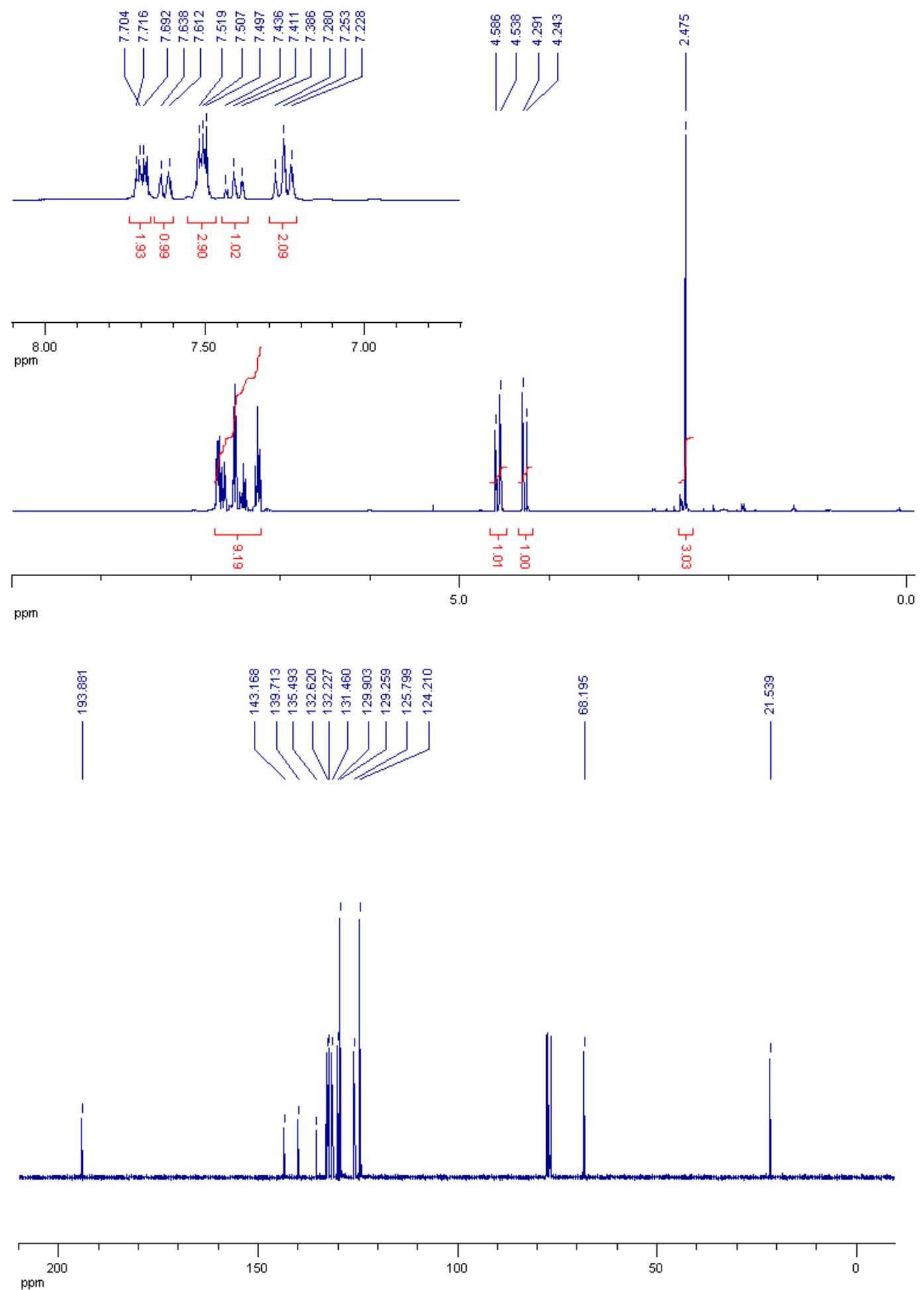


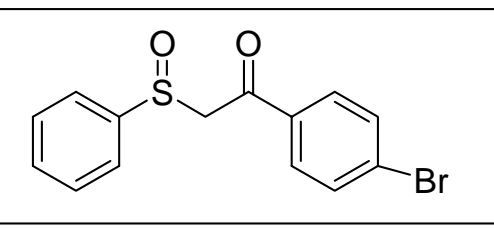

1-(4-bromophenyl)-2-(phenylsulfinyl)ethanone 3f.
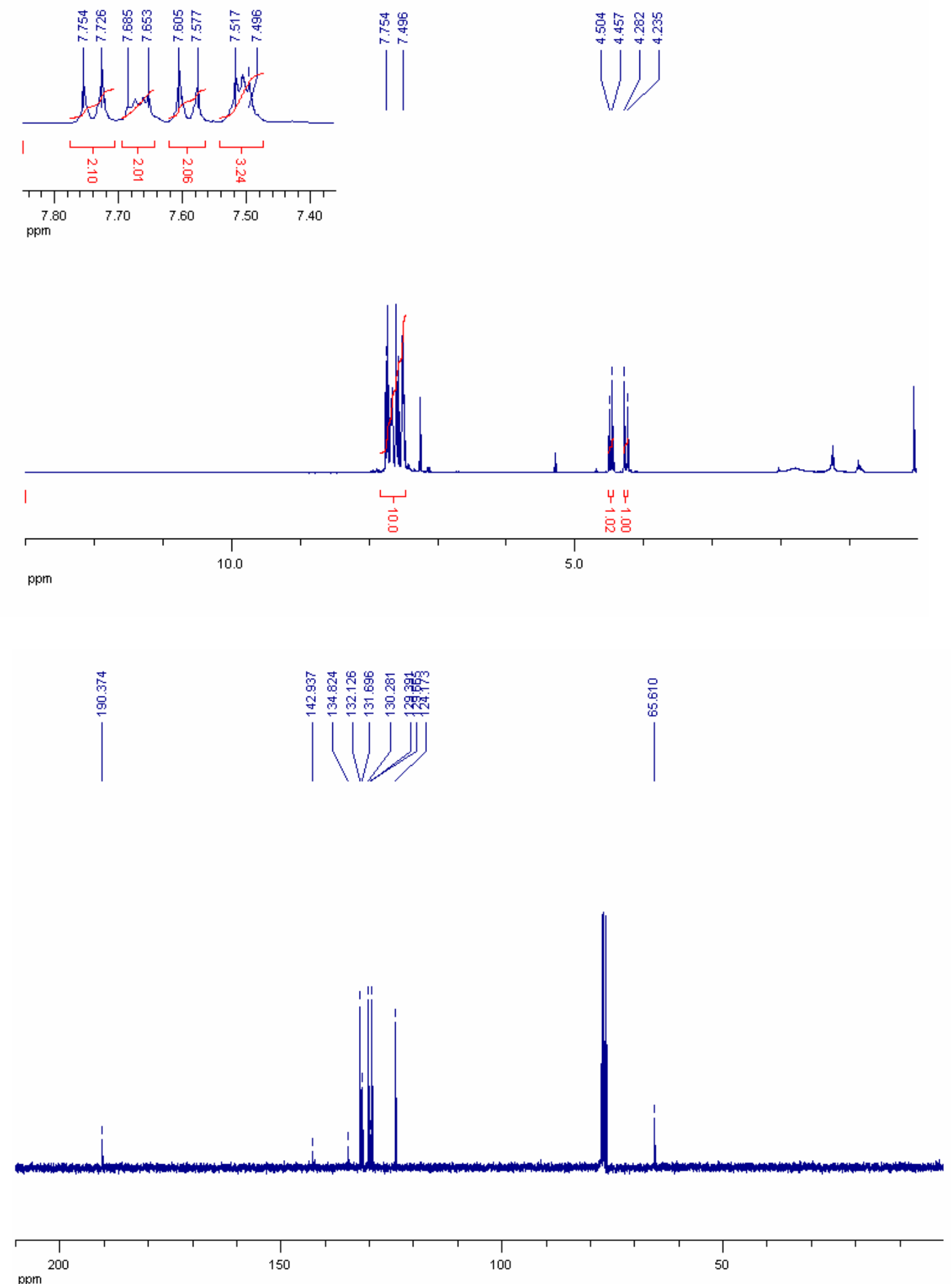


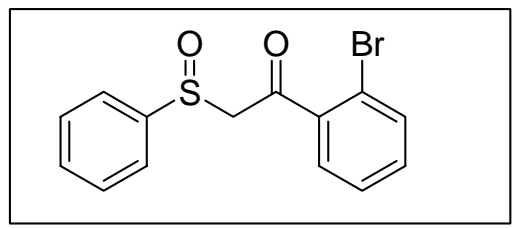

1-(2-bromophenyl)-2-(phenylsulfinyl)ethanone 3g.
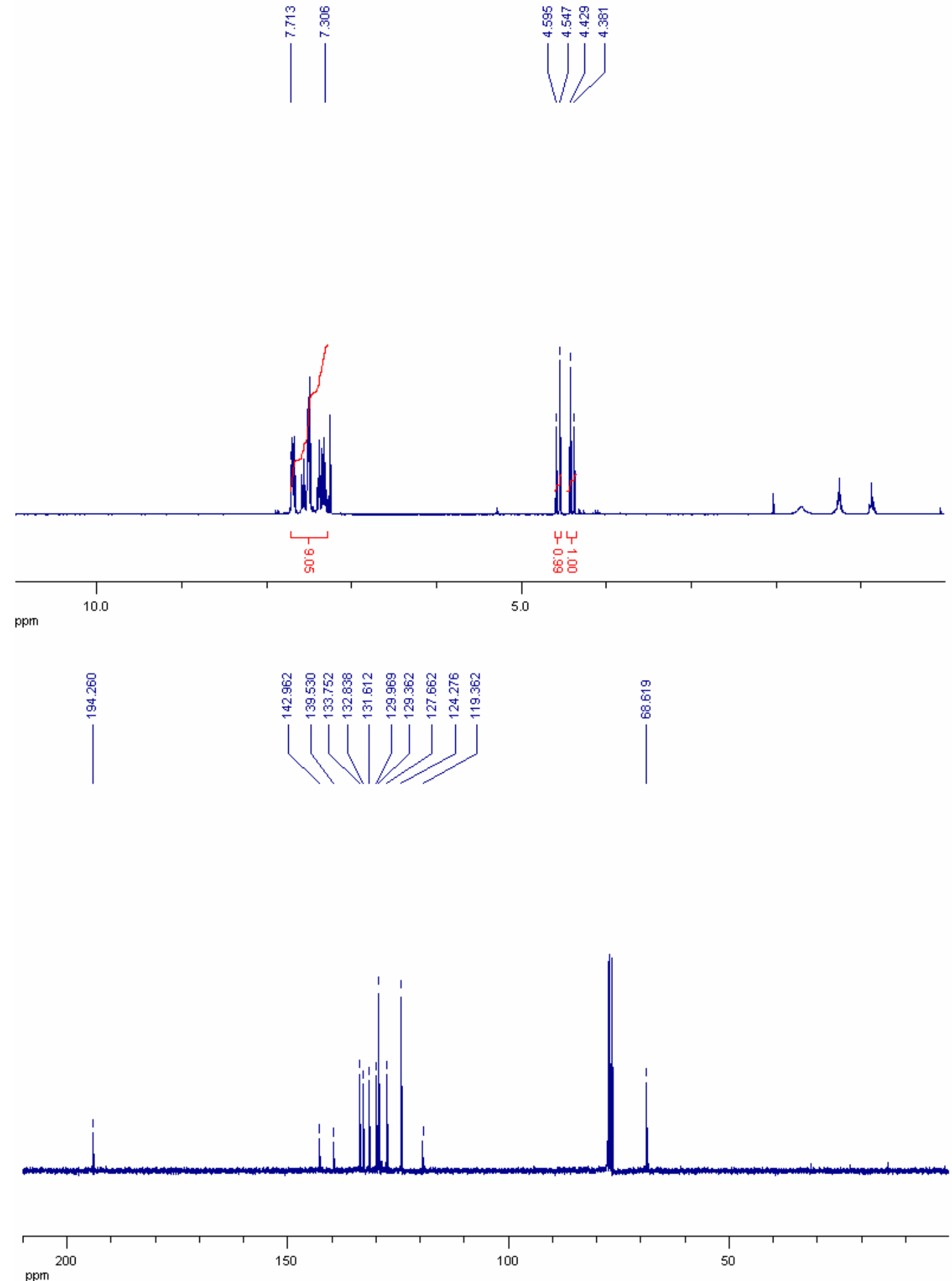


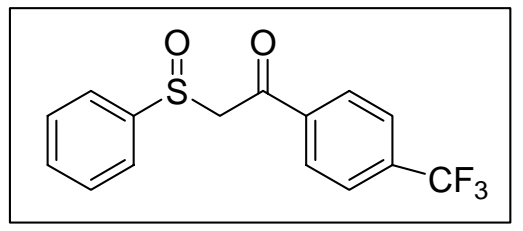

2-(phenylsulfinyl)-1-(4-(trifluoromethyl)phenyl)ethanone $\mathbf{3 h}$
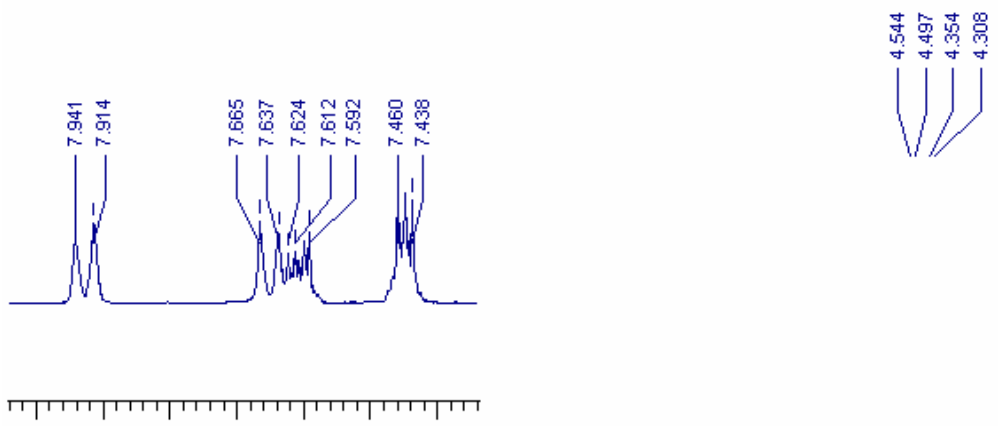

$\begin{array}{lllllll}8.00 & 7.90 & 7.80 & 7.70 & 7.60 & 7.50 & 7.40 \\ \text { ppm }\end{array}$
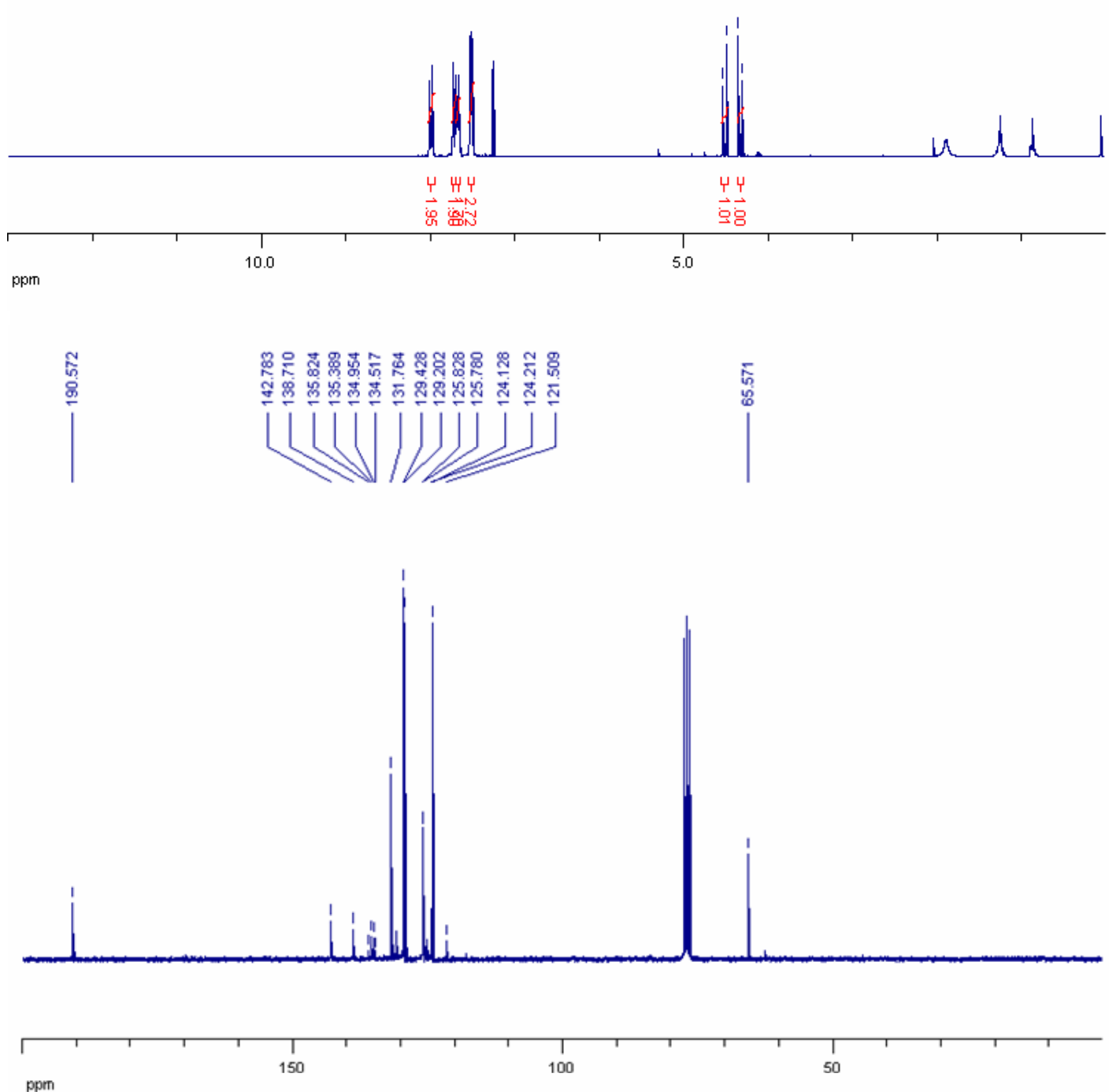


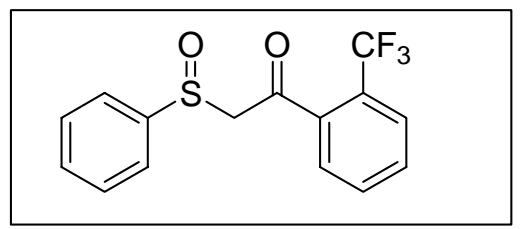

2-(phenylsulfinyl)-1-(2-(trifluoromethyl)phenyl)ethanone 3i
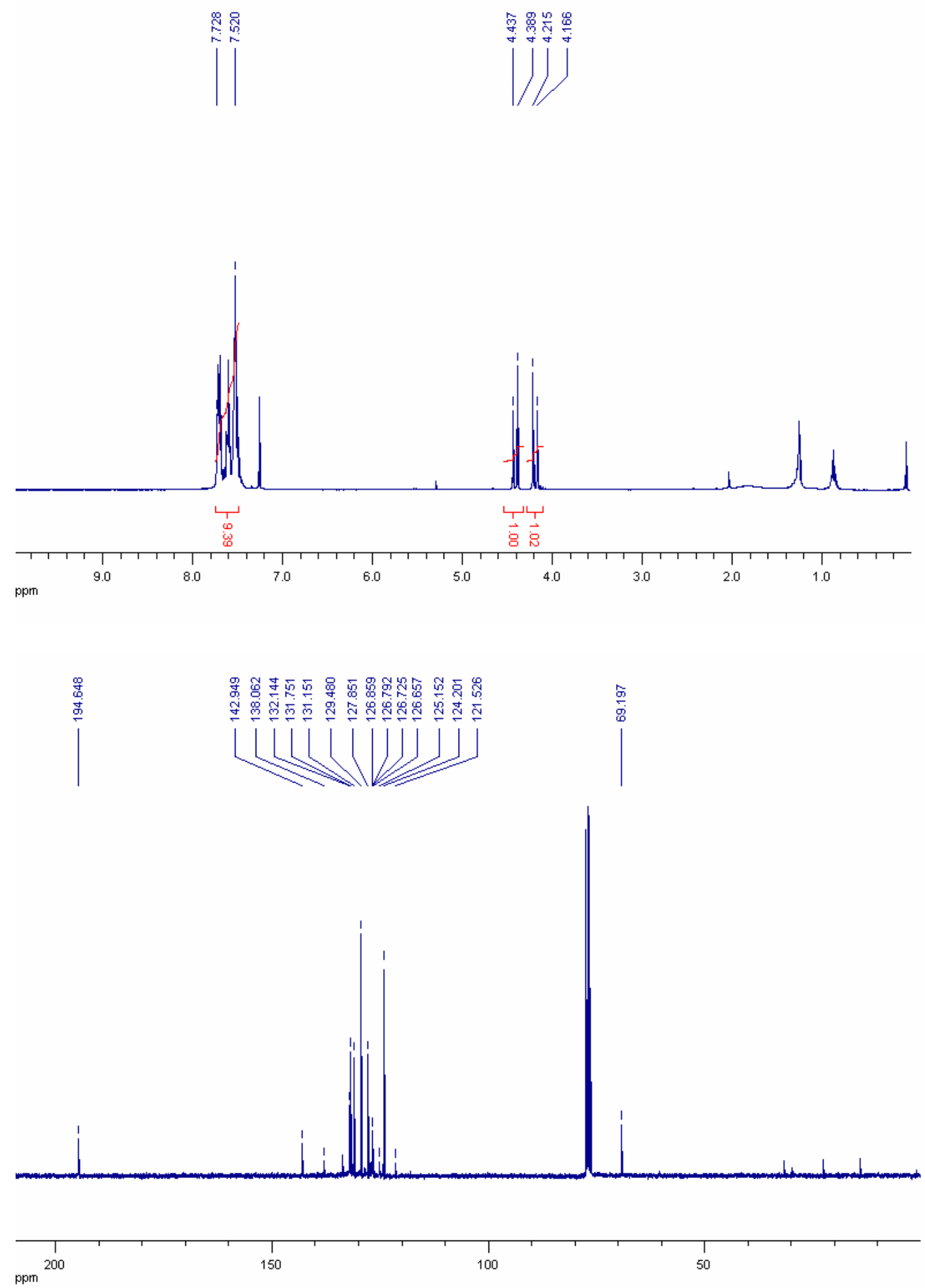


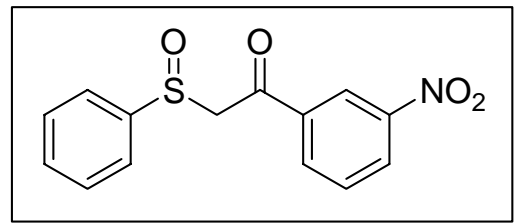

1-(3-nitrophenyl)-2-(phenylsulfinyl)ethanone 3k
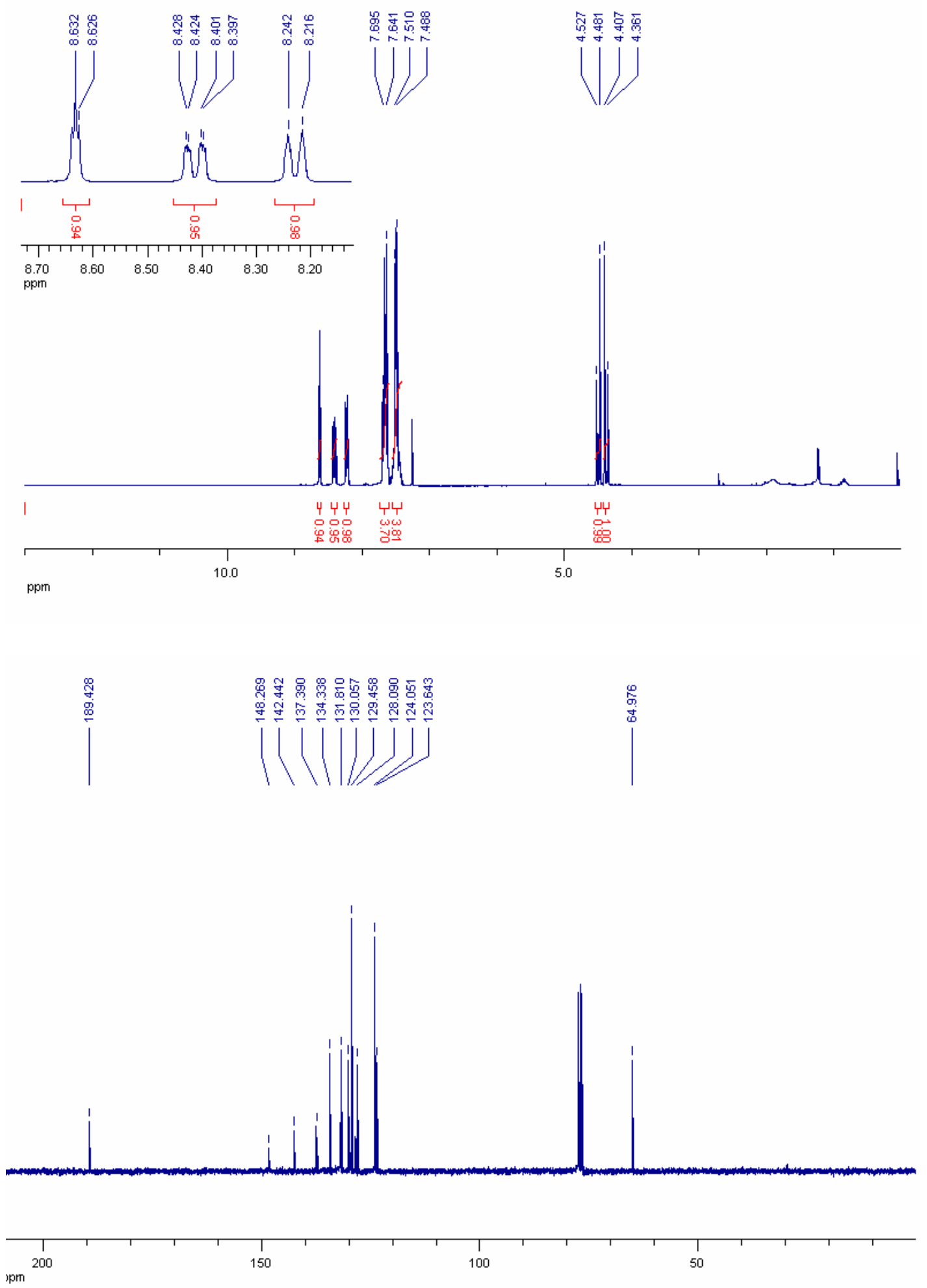


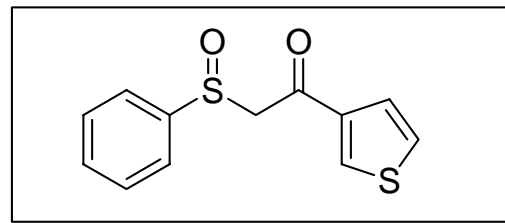

2-(phenylsulfinyl)-1-(3-thienyl)ethanone $3 \mathbf{l}$.
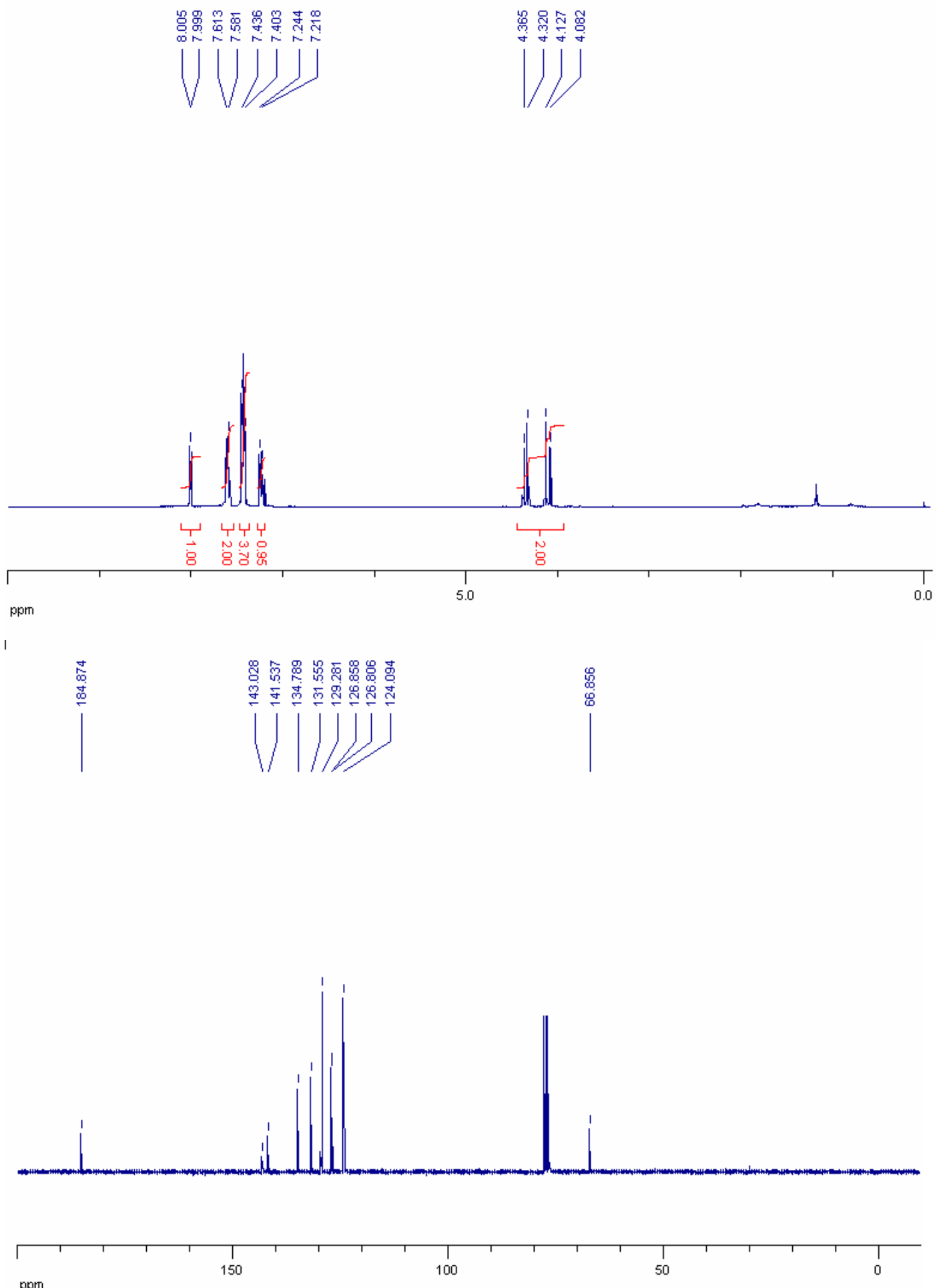


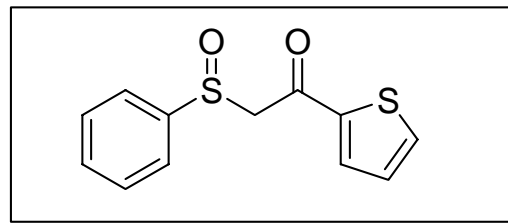

2-(phenylsulfinyl)-1-(2-thienyl)ethanone 3m.
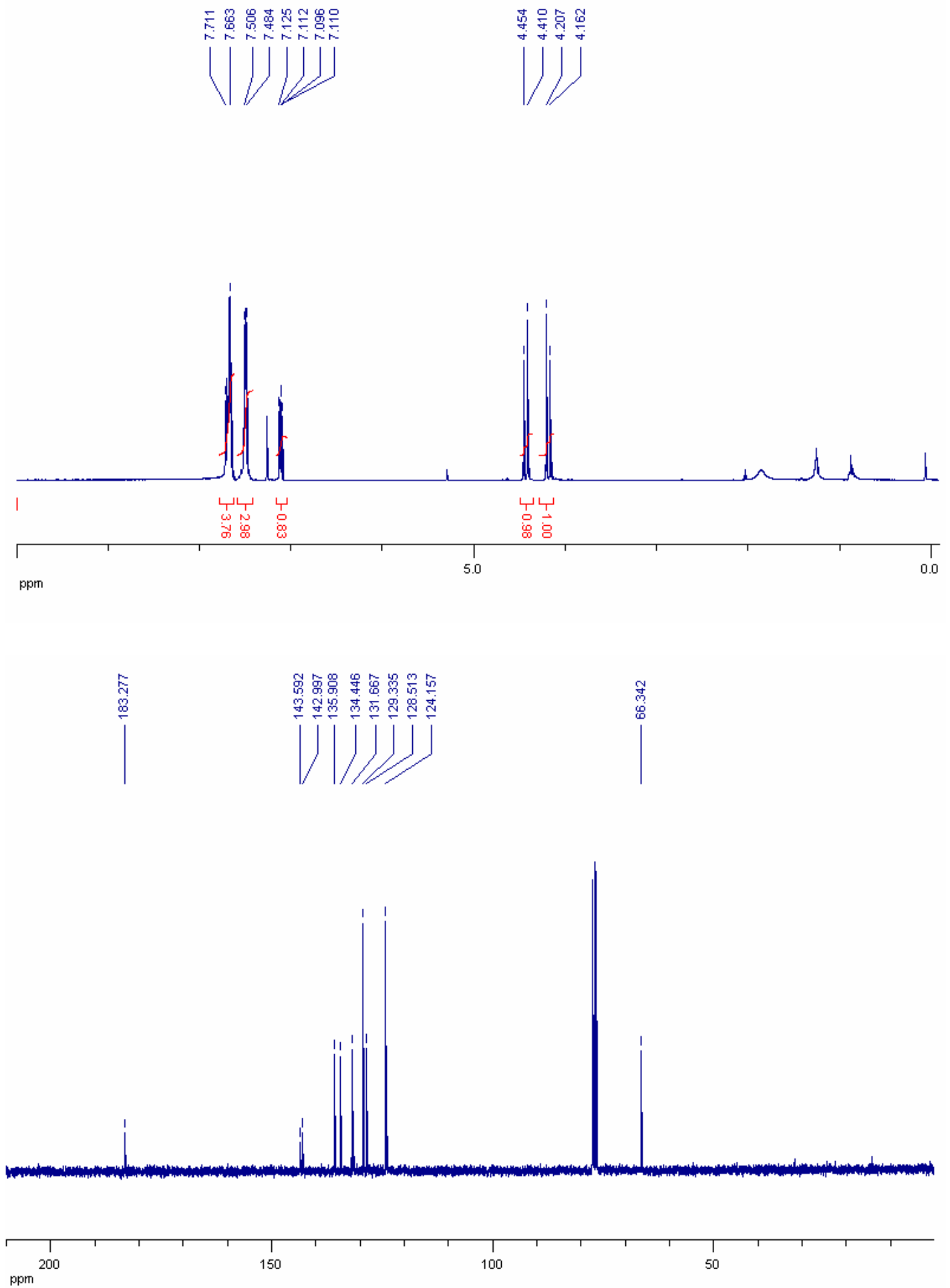


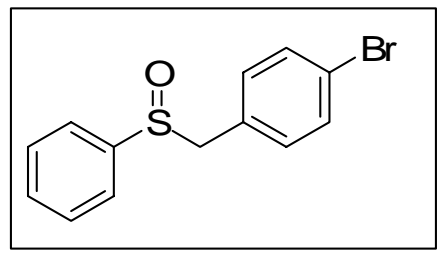

(4-bromobenzyl)phenyl sulfoxide $\mathbf{4 f}$

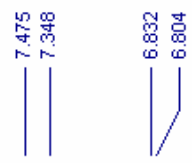

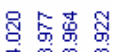

$(1)^{2}$
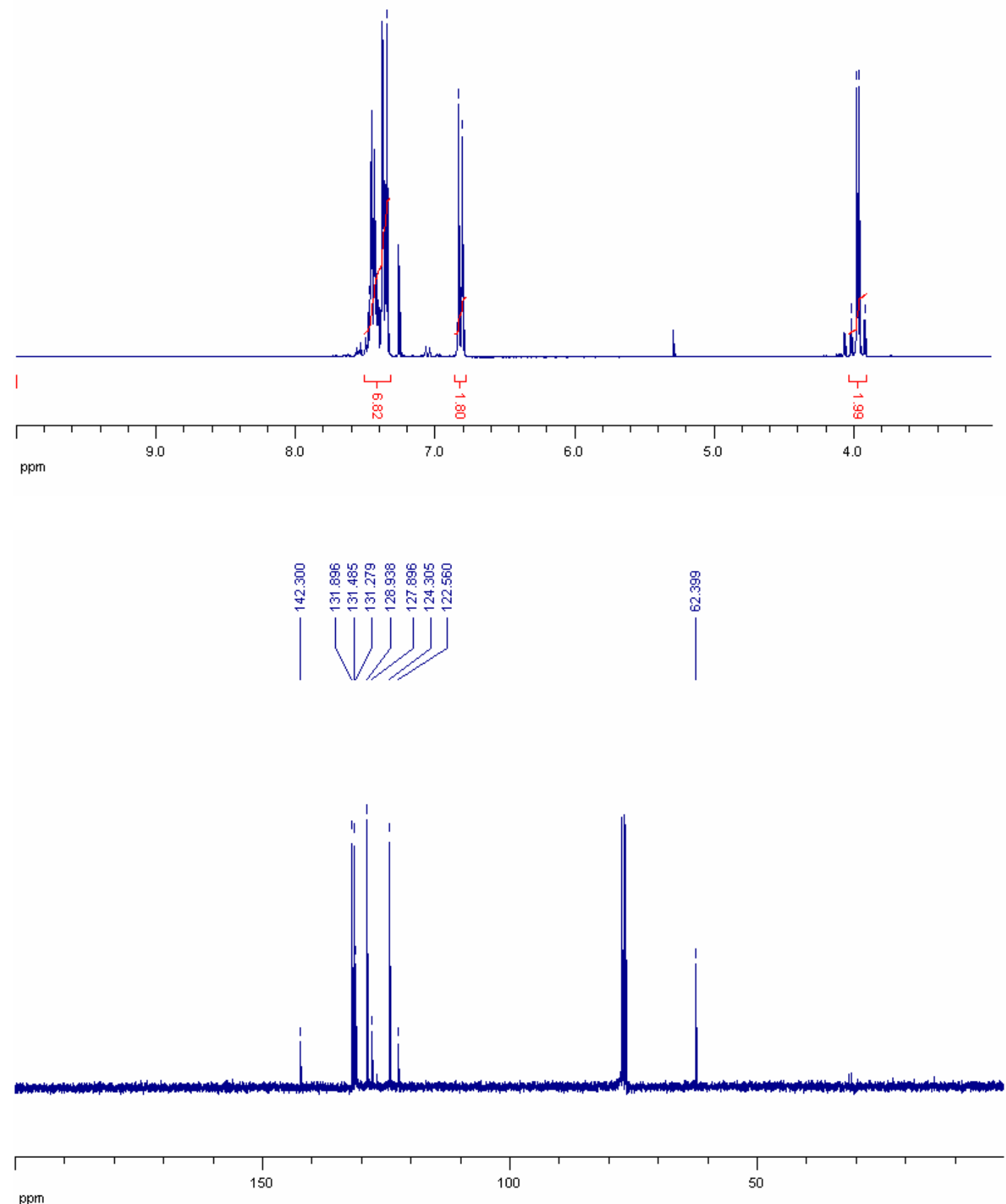


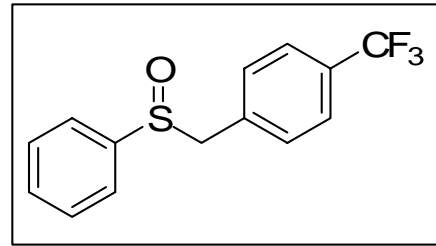

(4-trifluoromethylbenzyl)phenyl sulfoxide $\mathbf{4 h}$
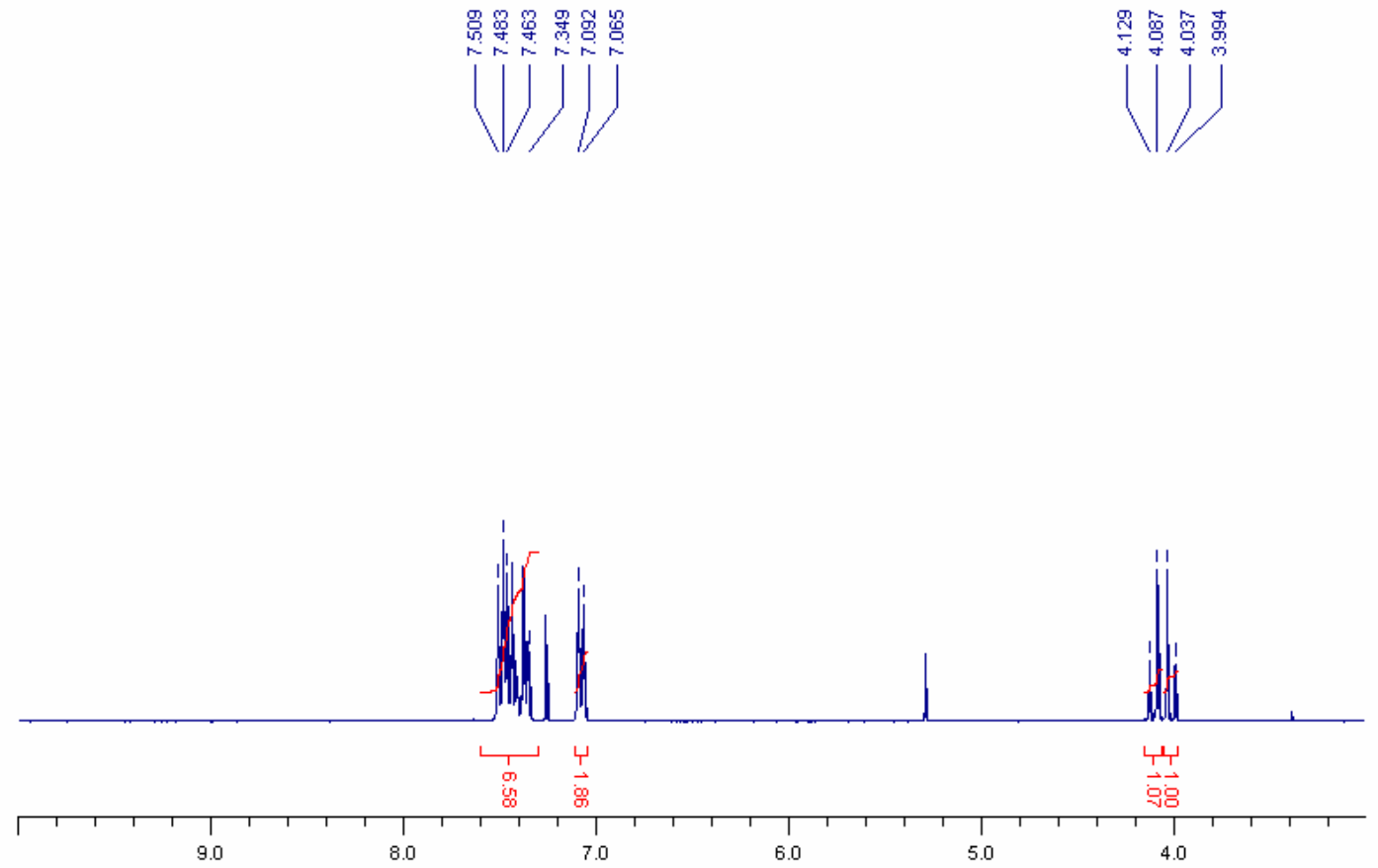

pprn

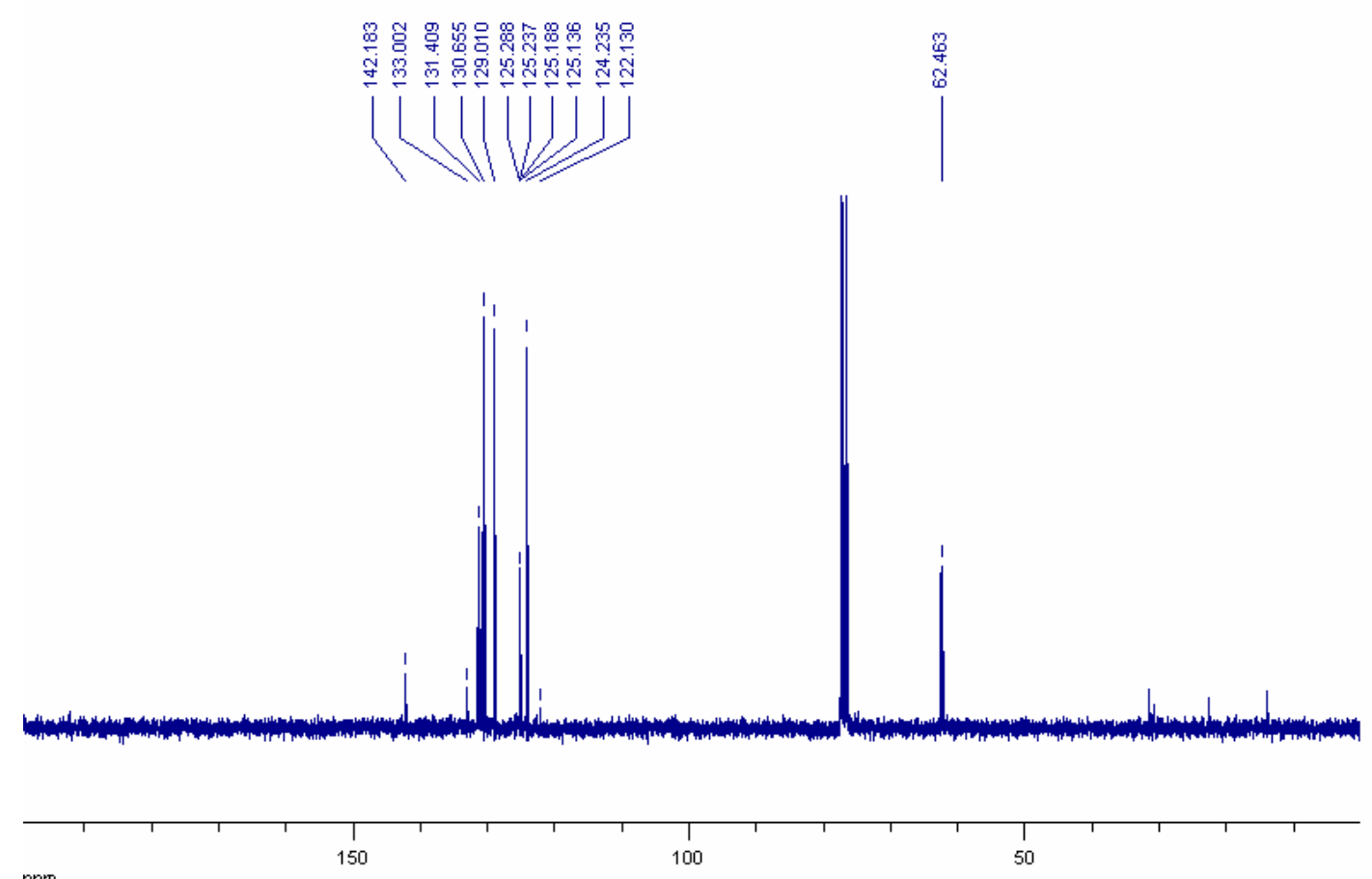

